\title{
Comparative modeling of class 1 lysyl tRNA synthetase from Treponema pallidum
}

\author{
Venkata Rao Dodaghatta Krishna Rao ${ }^{1}$, Rallapalli Ramanjeneyulu ${ }^{1}$, Dowlathabad Muralidhara Rao ${ }^{2^{*}}$ and \\ Chitta Suresh Kumar ${ }^{3}$ \\ ${ }^{1}$ Department of Microbiology, Sri Krishnadevaraya University, Anantapur, Andhra Pradesh, India; ${ }^{2}$ Department of \\ Biotechnology, Bioinformatics Laboratory, Sri Krishnadevaraya University, Anantapur; ${ }^{3}$ Department of Biochemistry, \\ Sri Krishnadevaraya University, Anantapur; \\ Dowlathabad Muralidhara Rao* - E-mail: muralidhararao@yahoo.co.in; * Corresponding author \\ received December 28, 2005; revised January 23, 2006; accepted January 24, 2006; published online January 24, 2006
}

\begin{abstract}
:
Lysyl tRNA synthetases facilitate amino acylation and play a crucial role in the essential cellular process of translation. They are grouped into two distinct classes (class I and class II). Class I lysyl tRNA synthetase is considered as a drug target for syphilis caused by Treponema pallidum. Comparative genome analysis shows the absence of its sequence homolog in eukaryotes. The structure of class I lysyl tRNA synthetase from Treponema pallidum is unknown and the difficulties in the in vitro culturing of Treponema makes it non-trivial. We used the structural template of class I lysyl tRNA synthetase from the archaea Pyrococcus horikoshii for modeling the Treponema pallidum lysyl tRNA synthetase structure. Thus, we propose the usefulness of the modeled class I lysyl tRNA synthetase for the design of suitable inhibitors towards the treatment of syphilis.
\end{abstract}

Keywords: Treponema pallidum; lysyl tRNA synthetase; modeller; syphilis; comparative modeling

\section{Background:}

Syphilis is a STD (sexually transmitted disease) caused by Treponema pallidum. The understanding of hostpathogen interactions remains unclear due to difficulty in culturing the organism in vitro. Syphilis is an exclusive human pathogen and its pathogenesis from animal study provides limited inference. [1] The availability of the complete genome sequence of Treponema pallidum provides ample opportunities for the computational analysis towards the identification of potential drug targets.

Lysyl tRNAs are essential for protein biosynthesis by ribosomal mRNA translation. They are synthesized by lysyl tRNA synthetases (a group of enzymes of two unrelated protein families, namely, class I and II). Known lysyl tRNA synthetases are class II in bacteria/eukaryotes and class I in archaea. A recent genomic analysis showed the presence of the archaeal type class-I lysyl tRNA synthetase in Treponema pallidum and Borellia species. [2] The difference between the lysyl tRNA synthetases of spirochetes and their hosts is for potential exploitation towards the development of anti-spirochete therapeutics. The X-ray crystal structure of class-I lysyl tRNA synthetase from the archaea Pyrococcus horikoshii is known. [3] Here, we use this structure as a template for the modeling of class-I lysyl tRNA synthetase from Treponema pallidum. We discuss the modeled structure as a potential drug target for syphilis caused by Treponema pallidum.

ISSN 0973-2063

Bioinformation 1(3): 81-82 (2006)
Methodology:

Lysyl tRNA synthetase sequence:

The protein sequence for class I lysyl tRNA synthetase of Treponema pallidum (O83650) was obtained from the SWISS PROT database.

\section{Template selection:}

The sequence was then searched against PDB (protein databank) protein sequences [4] using BLASTP [5] and a potential template structure (PDB-ID: 1IRX) was identified at an E-value (expect value) cut-off of 3e-110.

\section{Molecular modeling:}

An ensemble of 20 models for the class I lysyl tRNA synthetase from Treponema pallidum was generated using MODELLER by satisfaction of spatial restraints. [6]

\section{Model evaluation:}

The backbone of 20 models were overlapped well with the template structure and the most reliable structure was chosen based on least objective function value and stereo chemical quality of the models using the PROCHECK analysis. [7] In addition, the superimposition was also performed using the SUPERPOSE command in MODELLER and the RMSD (root mean square deviation) between predicted model and template is 0.7 angstrom. The predicted model quality assessment was checked using Ramachandran plot analysis for phi and psi torsion angles. The analysis shows that $92.5 \%$ of residues are found to be in the allowed region of the plot 


\title{
Bioinformation
}

\author{
www.bioinformation.net
}

by Biomedical Informatics Publishing Group

open access

which is more than the average cut-off of $90 \%$ in most reliable models. [8] The bond lengths and bond angles analyses of the modeled structure satisfies the small molecule experimental data described by Kabsch et al.,. [9]

Utility of the study:

Here, we illustrate the potential use of molecular models towards the likely design of inhibitors for the class I tRNA synthetase enzyme from Treponema pallidum. The study is highly pertinent where the difficulty of solving the structure of the protein is high (culturing of Treponema pallidum is difficult in this case).

\section{Conclusion:}

A molecular model of the class I lysyl tRNA synthetase from Treponema pallidum is documented in this study. The model is believed to provide valuable insights towards the design of an inhibitor for class I lysyl tRNA synthetase for the treatment of syphilis. However, further computational docking and high throughput screening

\section{Views \& Proposal}

experiments are required in detail for extracting more useful information.

\section{References:}

[1] R. W. Peeling, et al., Proc Natl. Acad. Sci., 94: 14383 (1997) [PMID:9405621]

[2] W. Hooke, J. Pathology, 208:224 (2006) [PMID: 16362988]

[3] Terada, et al., Nat. Struct Biol., 9:257 (2002) [PMID:11887185]

[4] http://www.rcsb.org/pdb/

[5] http://www.ncbi.nlm.nih.gov/blast/

[6] Sali \& T. L. Blundell, J. Mol. Biol., 234:779 (1993) [PMID:8254673]

[7] R. A. Laskowski, et al., J Appl Crystallogr., 26:283 (1993)

[8] G. N. Ramachandran, et al., J. Mol. Biol., 7:95 (1963) [PMID:13990617]

[9] W. Kabsch \& C. Sander, Biopolymers, 22:2577 (1983) [PMID:6667333]

Edited by N. Gautham

Citation: Rao et al., Bioinformation 1(3): 81-82 (2006)

License statement: This is an open-access article, which permits unrestricted use, distribution, and reproduction in any medium, for non-commercial purposes, provided the original author and source are credited. 